\title{
Guest Editor's Introduction: Special Issue on Workflow
}

\author{
Johan Montagnat • Ian J. Taylor
}

Published online: 9 August 2013

(C) Springer Science+Business Media Dordrecht 2013

This special issue of the Journal of Grid Computing on Workflows is a follow up to the 6th Workshop on Workflows in Support of LargeScale Science (WORKS'11) that was organised in conjunction with the SuperComputing conference in Seattle, November 2011. The papers collected in this issue are the result of an open call for extended or novel contributions on the workshop topics and a peer-review selection process. The call was very successful with 17 submissions out of which 11 have been selected for publication in this journal issue, showing the activity and the vivid interest of the scientific workflow research community.

In line with the series of WORKS workshops, WORKS'11 addressed the challenges related to the design of data-intensive (or scientific)

\footnotetext{
J. Montagnat $(\varangle)$

French National Centre for Scientific Research (CNRS), I3S/CNRS/UNS, Templiers building, 930 route des Colles, 06903 Sophia Antipolis Cedex, France

e-mail: johan.montagnat@cnrs.fr

I. J. Taylor

Computer Science \& Informatics, Cardiff University, Queen's Buildings, 5 The Parade, Roath, Cardiff CF24 3AA, UK

e-mail: Ian.J.Taylor@cs.cardiff.ac.uk
}

workflows and their enactment on large-scale distributed infrastructures in support of Big Science, including workflow execution in distributed environments, such as clusters, Grids and clouds, data intensive and data-driven workflows, execution performance, workflow monitoring for optimisation and troubleshooting, fault-tolerance and recovery techniques, etc. These traditional topics were well covered, with the four first papers in this issue addressing the problems of computing performance, optimisation, monitoring and reliability. Two novel themes that were introduced in the workshop were also well subscribed: workflow interoperability and workflow composition. Workflow interoperability is gaining attention in a context were many workflow representation languages and associated execution systems are in use, without a clearly prevalent standard emerging. Workflow composition is also becoming an important topic as workflow management systems are increasingly exploited by a larger end-users community, not necessarily well acquainted with distributed computing languages and techniques.

Four selected papers addressing workflow performance and reliability are first presented in this issue. The paper of J. Wozniak and co-authors addresses the problem of MPI-based parallel applications execution as part of Swift workflows enactment through a Many-ParallelTask-Computing paradigm. Y. Gu and co-authors 
also focus on workflow performance optimisation, targeting maximum throughput while executing on a faulty network through a bi-objective optimisation process. These two papers cover two aspects of execution performance arising in largescale environments: the efficient processing of parallel tasks and the protection against failures that necessarily happen. The two following papers by K. Vahi and co-authors, and V. Emeakahora and co-authors highlight the importance of workflow execution monitoring systems to improve performance and reliability. In the work of K. Vahi et al, a single monitoring system is integrated in multiple workflow enactors for real-time monitoring of workflow execution and troubleshooting. Conversely V. Emeakahora and co-authors use monitoring information to allocate cloud resources so as to optimise execution time.

Workflow interoperability is a theme common to the next four papers selected in this issue. Many scientific workflow systems have emerged during the last decade, and despite some laudable efforts no real consensus has emerged in this area, unlike the industry-driven BPEL standard widely adopted for business workflows. This fact is rather well recognised among the scientific workflow community, and usually not attributed to the lack of maturity of this domain alone, but also to the various aims followed by different systems. Yet, with the increasing adoption of workflow management systems to support large-scale scientific experiments, and the growing user communities behind workflow management environments, the need for interoperability among different systems arises.

Workflow interoperability can be implemented at different levels. When a community starts using different workflow systems, often comes a time when workflows written with different languages need to be exchanged, or even combined in a compound workflow with heterogeneous subcomponents. This is the problem addressed in the papers of K. Plankensteiner and co-authors on workflow languages interoperability and D. Rogers and coauthors on multi-workflow execution. The former work focuses on workflow code translation through a simple common interchange language, while the later describes self-contained executable workflow bundles, which can be used as building blocks for meta-workflows.

Workflows have a structuring role within user communities as a formal way of representing scientific experiments. Workflow interoperability is also considered as a means to share workflows within a community with a strong interest for domain-specific workflows reuse. This topic is covered in the works presented by A. LeBlanc and co-authors, and V. Korkhov and co-authors in the communities of heliophysics and neurosciences respectively. Both papers insist on the importance of rich annotation facilitating workflows reuse and integration in more complex computational processes. This momentum led to the emergence of platforms for multi-language workflow sharing over the last years, such as myExperiment and the SHIWA Simulation Platform, with particular focus on workflows documentation and multiworkflows execution respectively.

Finally, workflow composition is an increasingly investigated area as shown in the three last papers of this special issue. Workflow languages provide abstractions to describe complex interdependent tasks with asynchronous processing opportunities. The existing workflow languages and environments shield more or less end-users from the complexity of parallel programming and distributed enactment. Still, workflow modelling often requires a significant programming skill and the impact of the target distributed computing infrastructure should not be underestimated. Furthermore, the increasing use of workflow interoperability technologies within workflow user communities leads to workflow reuse (e.g. in meta-workflows) and repurposing needs for which workflow re-interpretation and re-edition are highly relevant. High-level abstraction frameworks and versatile workflow composition environments are developed to address these challenges. Ontology-based solutions, focusing on domain know-how, are described both in the work of G. Nadarajan and co-authors, in the context of video processing, and in the work of N. Cerezo and co-authors, in the context of medical imaging. A third paper by M. Sonntag and co-authors describe typical workflow composition shortcomings 
and propose an incremental workflow modelling platform that delivers high flexibility to workflow designers.

The Workshop on Workflows in Support of Large-Scale Science series of workshops is a very successful event organised each year in conjunction with the Supercomputing conference. The latest advances in this area will continue being presented at WORKS. We hope to meet you soon at WORKS. 